\title{
Analytical challenges of glycosaminoglycans at biological interfaces
}

\author{
Gergo Peter Szekeres ${ }^{1,2}\left(\mathbb{D} \cdot\right.$ Kevin Pagel $^{1,2} \mathbb{D} \cdot$ Zsuzsanna Heiner $^{3}(\mathbb{D}$
}

Received: 20 August 2021 / Revised: 24 September 2021 / Accepted: 28 September 2021 / Published online: 14 October 2021 (c) The Author(s) 2021

\begin{abstract}
The analysis of glycosaminoglycans (GAGs) is a challenging task due to their high structural heterogeneity, which results in diverse GAG chains with similar chemical properties. Simultaneously, it is of high importance to understand their role and behavior in biological systems. It has been known for decades now that GAGs can interact with lipid molecules and thus contribute to the onset of atherosclerosis, but their interactions at and with biological interfaces, such as the cell membrane, are yet to be revealed. Here, analytical approaches that could yield important knowledge on the GAG-cell membrane interactions as well as the synthetic and analytical advances that make their study possible are discussed. Due to recent developments in laser technology, we particularly focus on nonlinear spectroscopic methods, especially vibrational sum-frequency generation spectroscopy, which has the potential to unravel the structural complexity of heterogeneous biological interfaces in contact with GAGs, in situ and in real time.
\end{abstract}

Keywords Glycosaminoglycans $\cdot$ Lipids $\cdot$ Nonlinear spectroscopy $\cdot$ Sum-frequency generation spectroscopy $\cdot$ Interface/ surface analysis

\section{Analytical challenges of glycosaminoglycans}

Glycosaminoglycans (GAGs) are negatively charged, linear polysaccharides composed of repeating disaccharide building blocks containing a hexuronic acid and a hexosamine unit. Their negative charge originates from carboxylate groups in the hexuronic acids and sulfate groups at various positions of the hexuronic acid (2S) and the hexosamine (NS, 4S, 6S) moieties. The varying length of GAGs and their diverse sulfation patterns render their analysis a challenging task due to their

Published in the topical collection celebrating ABCs 20th Anniversary.

Kevin Pagel

kevin.pagel@fu-berlin.de

$\triangle$ Zsuzsanna Heiner

heinerzs@hu-berlin.de

1 Institut für Chemie und Biochemie, Freie Universität Berlin, Arnimallee 22, 14195 Berlin, Germany

2 Department of Molecular Physics, Fritz-Haber-Institut der Max-Planck-Gesellschaft, Faradayweg 4-6, 14195 Berlin, Germany

3 School of Analytical Sciences Adlershof, Humboldt-Universität zu Berlin, Albert-Einstein-Straße 5-11, 12489 Berlin, Germany high chemical and structural similarity. However, a correct and precise analysis of GAGs is crucial, which became especially apparent in 2008 in the Heparin Adulteration Crisis, in which heparin, a highly sulfated GAG used as an anticoagulant drug, was found to be contaminated with highly sulfated chondroitin sulfate, a structurally different GAG [1]. The administration of the adulterated heparin resulted in nearly a thousand health-related emergencies and deaths.

GAGs are usually located at the cell membrane, in the pericellular and extracellular matrix, and in granules within certain hematopoietic cells. This often means that GAGs are found at biological interfaces and barriers, which allow passage of specific small molecules, while larger molecules are retained at the exterior. These barriers can be covered by a layer called the glycocalyx, which in the past decade has gained increasing interest due to its prominent role in processes such as cancer development or drug delivery through the blood-brain-barrier [2]. Therefore, it is crucial to gain an in-depth knowledge about its constituents and their interaction at biological interfaces.

The analytical challenges of GAGs are a direct result of their structural diversity. GAGs are very abundant in the extracellular matrix (ECM) where they appear in free form or covalently attached to proteins as proteoglycans (PGs) (Figure 1). Each PG has one or more serine residues, which 


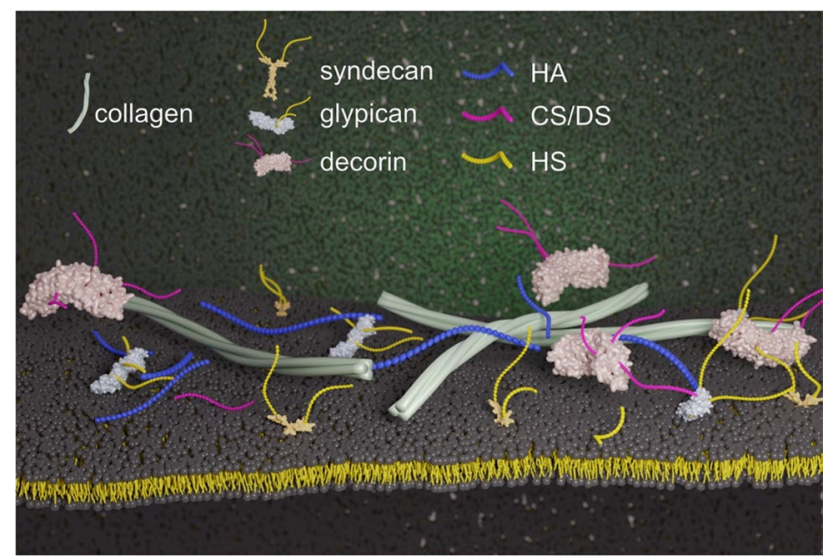

Fig. 1 Schematic representation of the extracellular matrix-cell membrane interface, where transmembrane (e.g., syndecan), membranebound (e.g., glypican), and free proteoglycans (e.g., decorin), as well as glycosaminoglycans (GAGs) can interact with collagen, membrane proteins, and the lipids of the cell membrane. The GAG chains of hyaluronic acid (HA), chondroitin/dermatan sulfate (CS/DS), and heparan sulfate (HS) are color-coded as blue, magenta, and yellow, respectively

each carry post-translationally attached GAG chains that determine the role of the PG in the organism. There are four distinct groups of GAGs: heparin (Hep)/heparan sulfate (HS), chondroitin sulfate (CS)/dermatan sulfate (DS), keratan sulfate (KS), and hyaluronic acid (HA). Out of these four groups, Hep/HS and CS/DS are the most complicated molecules, but also those that readily participate in a plethora of biological processes. In PGs, Hep/HS and CS/DS are covalently bound to a Ser residue via a xylosylated tetrasaccharide linker, while KS binds to $N$ - and $O$-glycans.

\section{Isolation and synthesis of GAGs}

In order to discuss the isolation and synthesis of GAGs, it is first crucial to understand the biological processes via which GAGs are biosynthesized. The biosynthesis of GAGs can be generally divided into two steps after the synthesis of the xylosylated tetrasaccharide linker on the specific Ser residues: the first step is the chain elongation, during which the hexuronic acid and hexosamine units are assembled into a chain [3]. In the second step, a variety of enzymes, such as deacetylases, sulfotransferases, and epimerases modify the chain with the possible removal of the acetyl group and the addition of sulfate groups in different positions in the disaccharide building blocks, thus producing a high variety of GAG molecules [3].

Naturally produced GAG chains, which are usually covalently bound to a proteoglycan, can be isolated either by beta elimination or by fully digesting the core protein of the proteoglycan, where the GAG chain is bound to a Ser residue via a tetrasaccharide linker. Once the protein digestion is complete, the GAG chain can be depolymerized by lyases and hydrolases. The choice of enzyme is crucial as the product of the depolymerization is enzyme-specific. It is possible to produce a wide range of different chain lengths by incomplete depolymerization, which in general falls in the degree of polymerization (dp) range of dp4-dp20. After the digestion and possible depolymerization, the GAGs undergo purification and enrichment, which often involves the use of an anion-exchange column or different chromatography techniques, such as size-exclusion chromatography and reverse-phase ion-pairing liquid chromatography [4].

The abovementioned techniques are useful tools to isolate GAGs from tissues that are rich in proteoglycans. However, the synthesis and selective sulfation of smaller GAG oligosaccharides has also been made possible by automated synthesis relying on specific blocks and the use of protective groups during polymerization [5]. More recently, the chemoenzymatic synthesis of GAGs involving the bacterial fermentation of the polysaccharide backbone and the subsequent sulfation with selective sulfotransferases allows for the higher yield production of GAGs, but this technique is generally used for the synthesis of highly sulfated chains, mostly Hep/HS [6, 7]. In addition, a library of genetically engineered cells which express differently modified GAG structures termed the GAGome was first reported in 2018, providing means to study the effects of these molecules under much more controlled circumstances than ever before [8].

\section{Interactions of GAGs}

\section{GAG-protein interactions}

Although GAGs play numerous roles in our body, including those in cancer, inflammation, embryonic development, or neuroplasticity, their interactions with other biomolecules pose as an exciting area of research [9]. GAGs are often found as polyanions at the cell membrane and in the extracellular matrix, which makes them prone to interact with other biomolecules, which carry positive charges or positively charged patches on their surfaces, such as proteins. The study of protein-GAG interactions, termed the $G A G$ interactome, has been a hot topic for the bioanalytical community for the past decades [10,11]. While heparin and its derivatives have long been used in hospitals as anti-coagulants, brought about by their interaction with antithrombin [12], and the role of Hep/HS in inducing cytokine storm has also been emergingly studied [13], the major focus of the scientific community has turned towards GAG-protein interactions in 2020, when it was found that HS plays an active role in the host infection of SARS-CoV-2 [14, 15]. 
To highlight the importance of protein-GAG interactions, a new database called MatrixDB has emerged in 2019 to provide a place to collect experimentally confirmed interactions between proteins and GAGs [16].

\section{GAG-lipid interactions}

The study of GAG-lipid interactions reaches back decades [17-24], when most studies in this area were performed to yield a better understanding on the role of GAGs in atherosclerosis. It was found that if the endothelium of the arteries is damaged, proteoglycans from the ECM can migrate into the arterial intima, where they can associate with lowdensity lipoproteins via a $\mathrm{Ca}^{2+}$ bridge [17]. This complex can then bind other lipophilic molecules, such as cholesterol. The continuous growth of this complex can lead to the formation of a plaque that protrudes into the arterial lumen, which alone can lead to insufficient blood flow; at the same time, the possibility of the rupture of the endothelium and the release of a fragment from this plaque can block the flow higher up the blood stream. By the same analogy, such an interaction has also been postulated to contribute to nanoplaque formation, a crucial step in the development of Alzheimer's disease [17].

However, there might be another aspect to lipid-GAG interactions. As mentioned above, GAGs are abundant in the peripheries of the cell membrane: they can be found in free form in the ECM, or bound to core proteins in ECM- or membrane-related proteoglycans. Therefore, their direct contact with the lipid moiety of the cell membrane is inevitable. Although, the lipid bilayer carries a neutral net surface charge in an environment with neutral $\mathrm{pH}$, it was found that the headgroups of zwitterionic phospholipids can carry a small net positive surface charge at (near-)neutral $\mathrm{pH}(7.0-7.4)[18,19]$, which can be the basis of interaction with the highly negatively charged GAGs. By discovering the interactions of GAGs with the lipid membrane, and the structure of GAGs at the membrane in situ, it will be possible to discuss important aspects of the role of GAGs and their structural properties in, e.g., drug-cell interactions and pathogen-cell infection mechanisms.

\section{Analytical approaches for GAG-lipid interactions}

In the past, especially before attempts made at producing homogeneous GAG chains with identical sulfation pattern were successful, most studies used dextran sulfate as a model molecule for GAGs. In general, GAG-lipid interactions can be observed by monitoring two parameters: (1) the changes of physical properties as a result of GAG-lipid association or (2) the changes in the structure of the lipid film or lipid vesicles due to the adsorption of GAG chains.

Early experiments relied on less complex instrumentation and studied the existence of interaction between GAGs and lipid vesicles. In such experiments, ultracentrifugation was used to precipitate the insoluble dextran sulfate-lipid complexes [20] or benefited from the drastic change of surface charge due to the association of the polyanion by studying changes in the electrophoretic mobility of the vesicles [21]. Soon after these studies, the importance of ${ }^{2} \mathrm{H}$ solid-state NMR increased: by quantitatively assessing the quadrupolar splitting of the $\alpha$ - and $\beta$-methylene peaks, researchers became able to assess how dextran sulfate interacts with multi-lamellar lipid vesicles [22]. These studies have been often augmented with other NMR techniques, e.g., magicangle spinning or nuclear Overhauser-enhanced spectroscopy [23], and solid-state structure characterization methods based on X-ray scattering[23, 24].

\section{Emerging bioanalytical tools for the characterization of GAG-lipid interactions}

In order to develop a better understanding of GAG-lipid interactions, it is crucial to perform experiments at physiologically relevant conditions. While solid-state approaches [22] (e.g., NMR) or gas-phase studies[25] (e.g., IR action spectroscopy) yield crucial structural information about homooligomeric complexes or molecules in isolation, it is challenging to study truly unaltered GAG-lipid interactions as these interactions in the body happen in solution and at the lipid-water interfaces.

An abundantly used analytical tool to study biomolecular changes and interactions under physiologically relevant conditions is fluorescence spectroscopy (e.g., super-resolution fluorescence microscopy, fluorescence lifetime imaging, fluorescence correlation spectroscopy, and two-photon fluorescence). From a physical-chemical point of view, however, the results from fluorescence may easily be biased due to the use of fluorescent labels. On the other hand, vibrational spectroscopic techniques such as Raman scattering and infrared (IR) spectroscopy are label-free, yet non-destructive tools to study the structure and chemical composition of matter. In comparison with vibrational spectroscopy, fluorescence spectroscopy lacks the chemical structural and compositional information, and therefore, it can only be an otherwise invaluable complementary tool to Raman scattering and IR spectroscopy. At the same time, normal Raman scattering and IR spectroscopy have their own limitations. GAG-lipid interactions naturally occur in an aqueous environment, which creates difficulties in IR (micro)spectroscopy experiments. With Raman scattering, homogeneous 
solutions of, e.g., different GAG molecules can be analyzed, but the obtained results may not be relevant on the cellular or tissue level, where both the GAG chains and the interacting partner, e.g., lipid membrane, are expected to be highly heterogeneous. Further difficulties are caused by the fact that even though a local enrichment of GAGs at the cell membrane is expected (either as free molecules or bound to a proteoglycan), their absolute concentration is still rather low-and often too low for normal Raman experiments. Further work is therefore required to overcome the problems and optimize the methodology to identify GAGs in live cells. Advances in surface-enhanced Raman scattering (SERS) can overcome some of the limitations of normal Raman experiments [26, 27]. SERS is well-known for the complex data sets collected from heterogeneous biomolecular systems, and studying a system with highly heterogeneous, yet chemically similar GAGs, the interpretation of the resulting data would require specialized data processing tools. The different ionic strength and $\mathrm{pH}$ in the extra- and intracellular matrices together with the highly negative charge of GAGs make the application of nanoparticles generally challenging. Furthermore, the question how the surface charge and chemical composition at interfaces with GAGs influence the nanoparticle uptake and drug delivery is yet to be answered [2].

When choosing the analytical method, one must also consider the sample type where GAG-lipid interactions could be studied informatively. The biological interfaces at which GAGs are expected to be particularly enriched (e.g., glycocalyx, cell membrane, and ECM) are highly heterogeneous and crowded biomolecular environments; therefore, we must select the simplest, yet still heterogeneous biomolecular system as a model interface to be studied by an analytical technique that provides the most detailed information about, e.g., the GAG-lipid interactions in real time.

When considering a spectroscopic tool for the study of real-time GAG-lipid interactions in a physiologically relevant environment, label-free and interface-selective techniques are needed. Such a tool should be sensitive enough to detect the concentration of the (heterogeneous) analyte far below the millimolar range while working in an aqueous environment with interface selectivity and/or sub-micrometer axial resolution. These challenging requirements are clearly fulfilled best by nonlinear spectroscopic techniques. In the following, nonlinear spectroscopic techniques applicable to study GAGlipid interactions are discussed in detail and summarized in Table 1.

\section{Coherent Raman scattering spectroscopy}

Coherent Raman scattering spectroscopy is a nonlinear technique based on Raman-active vibrational modes, which has evolved into two different techniques: coherent anti-Stokes 
Raman scattering (CARS) and stimulated Raman scattering (SRS). Both methods depend on a pump laser and a Stokes laser. The former is generally at a fixed wavelength while the latter is tuned to a frequency that is the difference of the pump frequency and the frequency of the molecular vibration of interest. These two laser beams are matched in the focus in both space and time, leading to a vibrational coherence, i.e., a vibrational population with phase-matched vibrations. In SRS, only the pump and the Stokes beams are used to generate the SRS signal, which is detected either as an increase in the measured intensity at the Stokes wavelength (stimulated Raman gain) or as an intensity loss at the pump wavelength (stimulated Raman loss). In CARS, also a third so-called probe beam is used, which excites the aforementioned vibrational population to another virtual vibrational state, from which the molecules relax back to the ground state, generating photons that are blue-shifted (e.g., to the anti-Stokes side of the spectrum) from the probe laser frequency by the frequency of the resonant molecular vibration. While coherent Raman scattering is not interface-selective, it is still a promising candidate to follow the assembly of lipids and GAGs [28], and its ability to provide three-dimensional information with spatial resolution at the order of $100 \mathrm{~nm}$ makes it an important tool [29]. These benefits have already been demonstrated when polysaccharide-protein interactions were studied with ${ }^{19} \mathrm{~F}$-labeled GAGs under physiological conditions using CARS [30]. The CARS microscopic analysis of GAG-lipid interactions in more complex samples, e.g., single cells or tissues can provide real-time three-dimensional chemical and compositional information about the enrichment of GAGs at the cell membrane.

\section{Second-harmonic generation spectroscopy}

Second-harmonic generation (SHG) spectroscopy is a second-order nonlinear optical tool, where high-intensity, femtosecond laser pulses at frequency $\omega$ are focused on the sample. Due to the induced second-order material polarization, two photons with the same $\omega$ frequency interact with the sample and a new photon is generated at the frequency of $2 \omega$. SHG is only generated in non-centrosymmetric media, which is fulfilled by highly ordered biomolecular structures. Furthermore, the symmetry is broken at any interface; therefore, molecular monolayers and their dynamics could be visualized by the surface-SHG technique. For example, real-time changes in protein conformation under dynamic conditions were recently studied in the presence of ligands and small molecules [31]. The biggest drawback of SHG is certainly that it generally does not provide structural information, but the technique allows for studying the orientation, conformation, symmetry, and disorder of biological macromolecules (e.g., collagen, myosin, tubulin, cellulose, and starch) and their assemblies in cells and tissues [32]. Furthermore, it is technically relatively straightforward to combine SHG imaging with other techniques such as twophoton fluorescence [33] or CARS microscopy for the collection of three-dimensional structural information in whole cells or tissues [28]. SHG can be useful for the simple determination of the higher order organization, conformation, and order-disorder of GAG chains at the lipid membrane even in real time. When SHG multiplexed with other microscopic techniques, it can provide information about both the interaction and the organization of GAG chains.

\section{Vibrational sum-frequency generation spectroscopy}

Fundamental understanding of the function-structure relations at biological interfaces and biological barriers is crucial where the local microenvironment and the intermolecular interactions can dictate and strongly modify this relation. Vibrational sum-frequency generation (VSFG) spectroscopy is an inherently interface-sensitive analytical tool that has a significant promise for studying the chemical structure, composition, and dynamics of molecules in situ and real time in a label-free manner at such interfaces. Similar to SHG, VSFG spectroscopy is also a second-order nonlinear method. The two incoming electromagnetic fields induce second-order material polarization, resulting in a new photon based on the sum-frequency generation process. Since centrosymmetry is broken at interfaces, the detection and chemical analysis of ordered molecular monolayers become possible even at very low surface coverages. During a broadband VSFG measurement, a narrowband, picosecond visible laser pulses $\left(\omega_{\mathrm{VIS}}\right)$ are temporally and spatially overlapping with a broadband, femtosecond mid-infrared laser pulses $\left(\omega_{\text {MIR }}\right)$ at the surface (Fig. 2a and b). The $\omega_{\text {MIR }}$ is resonant with the vibrational mode(s) of the investigated molecule(s). Through the sum-frequency generation process, the molecular vibrational fingerprint, generated by the $\omega_{\mathrm{MIR}}$, is up-converted with the help of the $\omega_{\mathrm{VIS}}$ to the visible spectral range (Fig $2 b$ ). The resulting VSFG spectra can be collected with a spectrometer equipped with a CCD. Applying different polarization combinations for each electromagnetic field involved in the SFG process (e.g., $p p p$ (or $s s p$ ), meaning $p(s)$ for VSFG, $p(s)$ for visible, and $p(p)$ for mid-infrared), the amplitudes, frequencies, and spectral widths of the given molecular vibrations can be determined. This polarization-sensitive nature of the technique, together with its interface specificity, enables the identification of molecular structures and orientations of chiral and achiral molecules and molecular groups at interfaces. The method can also work at any interfaces, such as air-water, oil-water, or buried interfaces, highlighting the application of VSFG spectroscopy for the investigation of bio-interfaces. Thanks 
Fig. 2 a Schematic representation of a VSFG experiment on a lipid monolayer-GAG system b with the Jablonski diagram of the VSFG process. c Average VSFG spectrum of a monolayer of the phospholipid, dipalmitoylphosphatidylcholine (DPPC), and its interaction with $\mathrm{CS}$ in the presence of $\mathrm{Ca}^{2+}$ ions at physiologically relevant concentrations. Figure adapted from ref. [39]
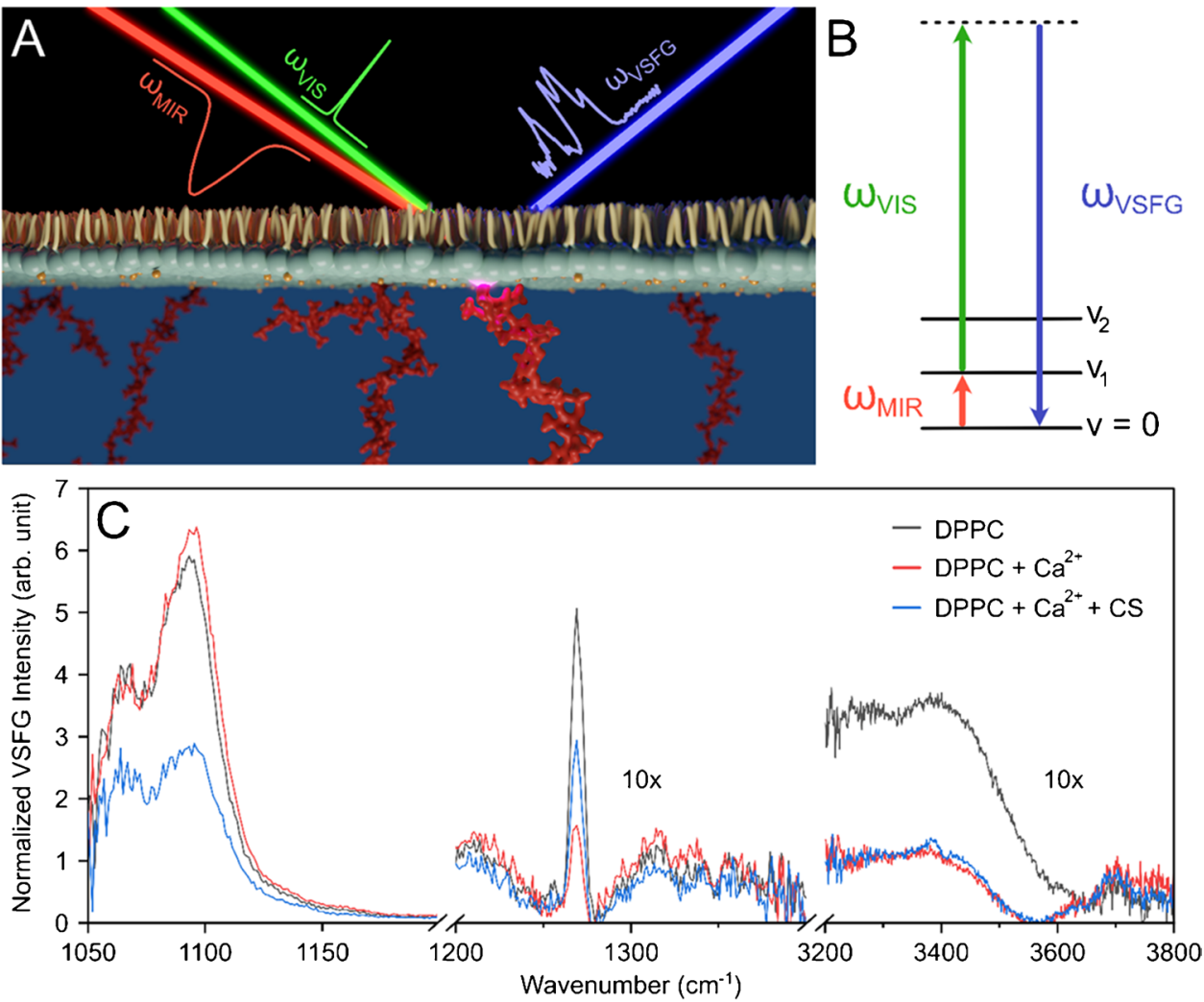

to the second-order SFG process, vibrational modes that are both IR- and Raman-active can be visualized.

In the last two decades, interfacial water structure and the orientation of lipid monolayers and asymmetric bilayers were thoroughly investigated by using achiral VSFG. On the one hand, this made it possible to understand the structural behavior of lipids during the hydration and their interaction with other biologically relevant molecules such as ions, charged small molecules, peptides, and proteins [34]. On the other hand, these studies helped to establish biologically relevant membrane model systems of different complexity for investigating real-time interactions at model membrane interfaces (e.g., lung surfactants) where the membrane fluidity and rigidity were monitored via the alkyl chain order. Due to the vast number of hydroxyl groups in GAGs and their different chemical environment as opposed to water molecules, changes in the hydration layer can be monitored simultaneously with changes in the chemical environment of GAGs at the interface.

In the past 20 years, studying buried polymer interfaces in situ by VSFG spectroscopy was also a focus in modern polymer science and chemical surface engineering, since the method gives interface-related structural information [35]. From the very beginning of the application of VSFG spectroscopy, the polymer-water interface was the most studied model system. The gained knowledge helped to design more hydrophilic and biocompatible polymers with favorable interfacial structure and composition for, e.g., body implants or drug delivery [36, 37]. All of these experiments conducted at aqueous interfaces will push forward the development of biocompatible and biodegradable polymers for biomedical and food sciences, creating a possibility to revolutionize our future. The development and optimization of analytical tools at such buried interfaces along the way will make it feasible to investigate the structurally highly heterogeneous GAGs at aqueous interfaces as well. For example, in a more recent study, the molecular structure, solvent interaction, and surface dynamics of hyaluronic acid were monitored at the air-water interface [38].

Many important biological molecules such as sugars, amino acids, or nucleic acids have one or more chiral centers. By condensation, these building blocks assemble into larger polysaccharides, proteins, or oligonucleotides, which in turn fold into chiral higher order structures. The development of chiral VSFG spectroscopy has revealed the secondary, tertiary, and higher order structures and orientations of various peptides and proteins in situ and real time [40]. These studies were conducted mainly at the air-water or even at a model membrane interface, allowing to study the folding-misfolding and aggregation of peptides and proteins and following their dynamics at a model membrane interface without dealing with the background signal of the membrane [41]. The advances in investigating the structure of proteins at the liquid, membrane, mineral, and synthetic 
interfaces were summarized recently highlighting the potential of VSFG spectroscopy in following the molecular-level spatiotemporal evolutions at bio-interfaces [42].

Recent developments in laser technology made it possible to increase the laser repetition rate employed in VSFG spectrometers by two orders of magnitude to $100 \mathrm{kHz}$ [43, 44], which led to a drastic increase in sensitivity and a shorter acquisition time ( $\leq 10 \mathrm{~s}$ ) for solid-supported heterogeneous phospholipid monolayers and bilayers $[45,46]$. This opens up a plethora of possibilities to study heterogeneous interfaces with increasing complexity in the future. The high sensitivity of the 100-kHz VSFG spectroscopy makes the study of molecular structural changes in, e.g., proteoglycans accessible upon their interaction with other molecules or saccharides with lipids [47].

In a proof-of-concept experiment series, we recently demonstrated that it is possible to study lipid-GAG interactions at the air-liquid interface within a DPPC monolayer on aqueous subphase containing $\mathrm{CS}$ and $\mathrm{Ca}^{2+}$ ions (Fig. 2c) at physiologically relevant salt- and sub-micromolar analyte concentrations [39]. During this interaction, the structural changes of the DPPC monolayer were monitored by VSFG spectroscopy. It was found that while the alkyl chains of lipids remain almost unchanged and the surface pressure decreased only few millinewtons per meter, the phosphate groups and the surrounding interfacial water molecules are highly affected by CS (see Fig. 2c). These results led to the conclusion that long CS chains form an organized layer below the DPPC monolayer, at which the CS aligns into a chiral secondary structure, most probably a helical coil [39]. The folding of GAG chains into helical segments was shown previously in solution, but has so far not been demonstrated at interfaces. In the future, with the advent of VSFG spectroscopy, more detailed information about the organization and the structure of GAG chains at model or cell membranes will be possible to be recovered.

While most traditional surface-sensitive techniques require high vacuum or surface selectivity via introducing inorganic interfaces, e.g., plasmonic nanoparticles, the VSFG technique can be directly applied to probe heterogeneous interfaces in situ and real time. Furthermore, such molecular structural information at bio-interfaces cannot be provided by using any other methods; therefore, VSFG spectroscopy will be one of the more powerful techniques to study heterogeneous bio-interfaces.

\section{Outlook}

Until now, spectroscopic experiments with model cell membranes were predominantly focused on proteins and lipids. However, when complex biological interfaces of true physiological relevance are addressed, it is crucial to also include
GAGs in the analysis. They are highly abundant at biological membranes and play a crucial role in essential processes at the interface. Currently, technologies to produce homogeneous GAG chains with identical sulfation patterns are emerging, which for the first time will enable a truly systematic assessment of the structure-function relationship of GAGs.

The effect of GAGs on the lipid membrane can be seen from two distinct points of view: (i) the primary effect is when the GAG chain directly interacts with the lipid head groups, which can lead to changes in the conformational order and membrane fluidity, while (ii) the secondary effect is observed during the interaction of proteins with the lipid membrane; here, the interaction can be drastically changed, e.g., by the electrostatic attachment of a polyanion.

GAG-lipid interactions are currently understudied; however, once these fundamental aspects are better understood, the knowledge on the role and structure of GAGs at the cell membrane and the ECM will grow exponentially. Currently, it is not understood how and to which extent GAG chains interact with the lipid membrane. This is further complicated when considering structural aspects. Even though an interaction between GAGs and lipids is expected, it is crucial to elucidate if it is functional or random, and whether a local enrichment of GAGs can be observed in regions where certain lipids are more abundant. Moreover, the interaction of the cell membrane with long GAG chains will likely influence the fluidity of the membrane and influence the uptake of certain cargo from the extracellular space. Changes in the membrane fluidity in close proximity of membrane-associated PGs can have further effect on cellular uptake or signaling. These are just a few examples, which once resolved will help to unravel the detailed molecular processes at the membrane-ECM interface.

Vibrational spectroscopy is a promising analytical tool to study GAG-membrane interactions. This especially applies to techniques that are based on nonlinear optical processes, as their selection rules can provide further benefits, e.g., vibrational information of enriched molecules in a threedimensional volume or high surface selectivity. With the recent advances in laser technology, broadband VSFG spectroscopy at a laser repetition rate of $\gg 10 \mathrm{kHz}$ has become a biocompatible and user-friendly technique allowing fast data acquisition even at sub-monolayer sensitivity. A range of experiments with increasing complexity of the model membrane will for example provide a better understanding of the dynamics and interactions at the cell membrane. Experiments addressing the structure and interaction of GAGs at the cell membrane will reveal the direct microenvironment of membrane PGs and how their GAG chains affect the fluidity of the cell membrane. Furthermore, these experiments will help to elucidate the role of the three-dimensional structure of the GAG chains on their interactions. However, systematic experiments of GAG-lipid interactions will require a 
suitable model system. Once the model system is optimized, highly homogeneous, long GAG chains can lead to a deeper understanding of the role of GAGs at the cell membraneextracellular matrix interface.

Acknowledgements Financial support for this research was provided by the German Research Foundation (Deutsche Forschungsgemeinschaft, DFG) Projektnummer 372486779-SFB 1340 to K.P., Projektnummer GSC 1013 SALSA to Z.H. and the European Union's Horizon 2020 Research and Innovation Programme grant number 899687-HSSEQ to K.P. and G.P.S. Z. H. further acknowledges the funding of her Julia Lermontova Fellowship by GSC 1013 SALSA.

Funding Open Access funding enabled and organized by Projekt DEAL.

\section{Declarations}

Conflict of interest The authors declare no competing interests.

Open Access This article is licensed under a Creative Commons Attribution 4.0 International License, which permits use, sharing, adaptation, distribution and reproduction in any medium or format, as long as you give appropriate credit to the original author(s) and the source, provide a link to the Creative Commons licence, and indicate if changes were made. The images or other third party material in this article are included in the article's Creative Commons licence, unless indicated otherwise in a credit line to the material. If material is not included in the article's Creative Commons licence and your intended use is not permitted by statutory regulation or exceeds the permitted use, you will need to obtain permission directly from the copyright holder. To view a copy of this licence, visit http://creativecommons.org/licenses/by/4.0/.

\section{References}

1. Guerrini M, Beccati D, Shriver Z, Naggi A, Viswanathan K, Bisio A, Capila I, Lansing JC, Guglieri S, Fraser B, Al-Hakim A, Gunay NS, Zhang Z, Robinson L, Buhse L, Nasr M, Woodcock J, Langer $\mathrm{R}$, Venkataraman $\mathrm{G}$, et al. Oversulfated chondroitin sulfate is a contaminant in heparin associated with adverse clinical events. Nat Biotechnol. 2008;266(26):669-75. https://doi.org/10.1038/ nbt1407.

2. Walter FR, Santa-Maria AR, Mészáros M, Veszelka S, Dér A, Deli MA. Surface charge, glycocalyx, and blood-brain barrier function. Tissue Barriers. 2021;1904773. https://doi.org/10.1080/21688 370.2021.1904773.

3. Pepi LE, Sanderson P, Stickney M, Amster IJ. Developments in mass spectrometry for glycosaminoglycan analysis: a review. Mol Cell Proteomics. 2021;20:100025. https://doi.org/10.1074/mcp. r120.002267.

4. Song Y, Zhang F, Linhardt RJ. Analysis of the glycosaminoglycan chains of proteoglycans. J Histochem Cytochem. 2020;69:121-35. https://doi.org/10.1369/0022155420937154.

5. Eller S, Collot M, Yin J, Hahm HS, Seeberger PH. Automated solid-phase synthesis of chondroitin sulfate glycosaminoglycans. Angew Chemie Int Ed. 2013;52:5858-61. https://doi.org/10.1002/ ANIE. 201210132.

6. Li J, Su G, Liu J. Enzymatic synthesis of homogeneous chondroitin sulfate oligosaccharides. Angew Chemie Int Ed. 2017;56:11784-7. https://doi.org/10.1002/ANIE.201705638.
7. Lu W, Zong C, Chopra P, Pepi LE, Xu Y, Amster IJ, Liu J, Boons G-J. Controlled chemoenzymatic synthesis of heparan sulfate oligosaccharides. Angew Chemie Int Ed. 2018;57:5340-4. https:// doi.org/10.1002/ANIE.201800387.

8. Chen YH, Narimatsu Y, Clausen TM, Gomes C, Karlsson R, Steentoft C, Spliid CB, Gustavsson T, Salanti A, Persson A, Malmström A, Willén D, Ellervik U, Bennett EP, Mao Y, Clausen $\mathrm{H}$, Yang $\mathrm{Z}$. The GAGOme: a cell-based library of displayed glycosaminoglycans. Nat Methods. 2018;15:881-8. https://doi.org/ 10.1038/s41592-018-0086-z.

9. Xu D, Esko JD. Demystifying heparan sulfate-protein interactions. Annu Rev Biochem http://dx.doi.org/101146/annurev-bioch em-060713-035314. 2014;83:129-57. https://doi.org/10.1146/ ANNUREV-BIOCHEM-060713-035314.

10. Gómez Toledo A, Sorrentino JT, Sandoval DR, Malmström J, Lewis NE, Esko JD. A systems view of the heparan sulfate interactome. J Histochem Cytochem. 2021;69:105-19.

11. Capila I, Linhardt RJ. Heparin - protein interactions. Angew Chemie - Int Ed. 2002;41:390-412.

12. Petitou M, Casu B, Lindahl U. 1976-1983, a critical period in the history of heparin: the discovery of the antithrombin binding site. Biochimie. 2003;85:83-9.

13. Cai Z, Yarovoi SV, Zhu Z, Rauova L, Hayes V, Lebedeva T, Liu Q, Poncz M, Arepally G, Cines DB, Greene MI. Atomic description of the immune complex involved in heparin-induced thrombocytopenia. Nat Commun. 2015;6:1-10. https://doi.org/ 10.1038/ncomms9277.

14. Clausen TM, Sandoval DR, Spliid CB, Pihl J, Perrett HR, Painter CD, Narayanan A, Majowicz SA, Kwong EM, McVicar RN, Thacker BE, Glass CA, Yang Z, Torres JL, Golden GJ, Bartels PL, Porell RN, Garretson AF, Laubach L, et al. SARSCoV-2 infection depends on cellular heparan sulfate and ACE2. Cell. 2020;183:1043-1057.e15. https://doi.org/10.1016/j.cell. 2020.09.033.

15. Nie C, Pouyan P, Lauster D, Trimpert J, Kerkhoff Y, Szekeres GP, Wallert M, Block S, Sahoo AK, Dernedde J, Pagel K, Kaufer BB, Netz RR, Ballauff M, Haag R. Polysulfates Block SARS-CoV-2 uptake through electrostatic interactions**. Angew Chemie Int Ed. 2021;60:15870-8. https://doi.org/10. 1002/ANIE.202102717.

16. Clerc O, Deniaud M, Vallet SD, Naba A, Rivet A, Perez S, Thierry-Mieg N, Ricard-Blum S. MatrixDB: integration of new data with a focus on glycosaminoglycan interactions. Nucleic Acids Res. 2019;47:D376-81. https://doi.org/10.1093/nar/gky10 35.

17. Siegel G, Mockenhaupt FHME, Behnke AL, Ermilov E, Winkler K, Pries AR, Malmsten M, Hetzer R, Saunders R, Lindman B. Lipoprotein binding to anionic biopolyelectrolytes and the effect of glucose on nanoplaque formation in arteriosclerosis and Alzheimer's disease. Adv Colloid Interface Sci. 2016;232:25-35.

18. Klausen LH, Fuhs T, Dong M. Mapping surface charge density of lipid bilayers by quantitative surface conductivity microscopy. Nat Commun. 2016;7:1-10. https://doi.org/10.1038/ncomms12447.

19. Fuhs T, Klausen LH, Sønderskov SM, Han X, Dong M. Direct measurement of surface charge distribution in phase separating supported lipid bilayers. Nanoscale. 2018;10:4538-44. https://doi. org/10.1039/c7nr09522h.

20. Kim YC, Nishida T. Nature of interaction of dextran sulfate with lecithin dispersions and lysolecithin micelles. J Biol Chem. 1977;252:1243-9. https://doi.org/10.1016/s0021-9258(17) 40647-8.

21. Krumbiegel M, Arnold K. Microelectrophoresis studies of the binding of glycosaminoglycans to phosphatidylcholine liposomes. Chem Phys Lipids. 1990;54:1-7. https://doi.org/10.1016/00093084(90)90053-T. 
22. Huster D, Arnold K. Ca2+-mediated interaction between dextran sulfate and dimyristoyl-sn- glycero-3-phosphocholine surfaces studied by $2 \mathrm{H}$ nuclear magnetic resonance. Biophys J. 1998;75:909-16. https://doi.org/10.1016/S0006-3495(98) 77579-4.

23. Huster D, Dietrich U, Gutberlet T, Gawrisch K, Arnold K. Lipid matrix properties in cationic membranes interacting with anionic polyelectrolytes: a solid-state NMR approach. Langmuir. 2000;16:9225-32. https://doi.org/10.1021/la000620d.

24. De Meijere K, Brezesinski G, Zschörnig O, Arnold K, Möhwald H. Structure studies of a phospholipid monolayer coupled to dextran sulfate. Phys B Condens Matter. 1998;248:269-73. https:// doi.org/10.1016/S0921-4526(98)00246-4.

25. Lettow M, Grabarics M, Mucha E, Thomas DA, Polewski $Ł$, Freyse J, Rademann J, Meijer G, von Helden G, Pagel K. IR action spectroscopy of glycosaminoglycan oligosaccharides. Anal Bioanal Chem. 2020;412:533-7. https://doi.org/10.1007/ s00216-019-02327-7.

26. Szekeres GP, Kneipp J. SERS probing of proteins in gold nanoparticle agglomerates. Front Chem. 2019;7. https://doi.org/10.3389/ fchem.2019.00030.

27. Kneipp J, Kneipp H, McLaughlin M, Brown D, Kneipp K. In vivo molecular probing of cellular compartments with gold nanoparticles and nanoaggregates. Nano Lett. 2006;6:2225-31. https://doi. org/10.1021/n1061517x.

28. Moura CC, Bourdakos KN, Tare RS, Oreffo ROC, Mahajan S. Live-imaging of bioengineered cartilage tissue using multimodal non-linear molecular imaging. Sci Rep. 2019;9:1-9. https://doi. org/10.1038/s41598-019-41466-w.

29. Li S, Li Y, Yi R, Liu L, Qu J (2020) Coherent anti-Stokes Raman scattering microscopy and its applications. Front Phys 0:515. https://doi.org/10.3389/FPHY.2020.598420

30. Lima MA, Cavalheiro RP, Viana GM, Meneghetti MCZ, Rudd TR, Skidmore MA, Powell AK, Yates EA. 19F labelled glycosaminoglycan probes for solution NMR and non-linear (CARS) microscopy. Glycoconj J. 2017;34:405-10. https://doi.org/10. 1007/s10719-016-9723-x.

31. Donohue E, Khorsand S, Mercado G, Varney KM, Wilder PT, Yu W, MacKerell AD, Alexander P, Van QN, Moree B, Stephen AG, Weber DJ, Salafsky J, McCormick F. Second harmonic generation detection of Ras conformational changes and discovery of a small molecule binder. Proc Natl Acad Sci U S A. 2019;116:17290-7. https://doi.org/10.1073/pnas.1905516116.

32. Brasselet S. Polarization-resolved nonlinear microscopy: application to structural molecular and biological imaging. Adv Opt Photonics. 2011;3:205. https://doi.org/10.1364/aop.3.000205.

33. Heiner Z, Zeise I, Elbaum R, Kneipp J. Insight into plant cell wall chemistry and structure by combination of multiphoton microscopy with Raman imaging. J Biophotonics. 2018;11:e201700164. https://doi.org/10.1002/JBIO.201700164.

34. Jubb AM, Hua W, Allen HC. Environmental chemistry at vapor/ water interfaces: insights from vibrational sum frequency generation spectroscopy. Annu Rev Phys Chem. 2012;63:107-30. https:// doi.org/10.1146/annurev-physchem-032511-143811.

35. Lu X, Zhang C, Ulrich N, Xiao M, Ma YH, Chen Z. Studying polymer surfaces and interfaces with sum frequency generation vibrational spectroscopy. Anal. Chem. 2017;89:466-89.
36. Oda Y, Horinouchi A, Kawaguchi D, Matsuno H, Kanaoka S, Aoshima S, Tanaka K. Effect of side-chain carbonyl groups on the interface of vinyl polymers with water. Langmuir. 2014;30:12159. https://doi.org/10.1021/la404802j.

37. Yang F, Zhang X, Song L, Cui H, Myers JN, Bai T, Zhou Y, Chen $\mathrm{Z}, \mathrm{Gu}$ N. Controlled drug release and hydrolysis mechanism of polymer-magnetic nanoparticle composite. ACS Appl Mater Interfaces. 2015;7:9410-9. https://doi.org/10.1021/acsami.5b02210.

38. Moll CJ, Giubertoni G, Versluis J, Koenderink GH, Bakker HJ. Molecular structure and surface accumulation dynamics of hyaluronan at the water-air interface. Macromolecules. 2021;54:865563. https://doi.org/10.1021/acs.macromol.1c00366.

39. Szekeres GP, Krekic S, Miller RL, Mero M, Pagel K, Heiner Z. The interaction of chondroitin sulfate with a lipid monolayer observed by using nonlinear vibrational spectroscopy. Phys Chem Chem Phys. 2021;23:13389-95. https://doi.org/10.1039/d1cp0 1975a.

40. Yan ECY, Fu L, Wang Z, Liu W. Biological macromolecules at interfaces probed by chiral vibrational sum frequency generation spectroscopy. Chem Rev. 2014;114:8471-98.

41. Fu L, Ma G, Yan ECY. In situ misfolding of human islet amyloid polypeptide at interfaces probed by vibrational sum frequency generation. J Am Chem Soc. 2010;132:5405-12. https://doi.org/ 10.1021/ja909546b.

42. Hosseinpour S, Roeters SJ, Bonn M, Peukert W, Woutersen S, Weidner T. Structure and dynamics of interfacial peptides and proteins from vibrational sum-frequency generation spectroscopy. Chem Rev. 2020;120:3420-65.

43. Heiner Z, Petrov V, Mero M. Compact, high-repetition-rate source for broadband sum-frequency generation spectroscopy. APL Photonics. 2017;2:066102. https://doi.org/10.1063/1.4983691.

44. Heiner Z, Wang L, Petrov V, Mero M. Broadband vibrational sum-frequency generation spectrometer at $100 \mathrm{kHz}$ in the 950 $1750 \mathrm{~cm}-1$ spectral range utilizing a LiGaS 2 optical parametric amplifier. Opt Express. 2019;27:15289. https://doi.org/10.1364/ OE.27.015289.

45. Yesudas F, Mero M, Kneipp J, Heiner Z. Vibrational sum-frequency generation spectroscopy of lipid bilayers at repetition rates up to $100 \mathrm{kHz}$. J Chem Phys. 2018;148:104702. https://doi.org/ 10.1063/1.5016629.

46. Yesudas F, Mero M, Kneipp J, Heiner Z. High-resolution and high-repetition-rate vibrational sum-frequency generation spectroscopy of one- and two-component phosphatidylcholine monolayers. Anal Bioanal Chem. 2019;411:4861-71. https://doi.org/ 10.1007/s00216-019-01690-9.

47. Link KA, Hsieh CY, Tuladhar A, Chase Z, Wang Z, Wang H, Walker RA. Vibrational studies of saccharide-induced lipid film reorganization at aqueous/air interfaces. Chem Phys. 2018;512:104-10. https://doi.org/10.1016/j.chemphys.2018.02. 011.

Publisher's note Springer Nature remains neutral with regard to jurisdictional claims in published maps and institutional affiliations. 